\title{
Dem Wahnsinn Einhalt gebieten? - Die aktuelle US-amerikanische Leitlinie zur Senkung der Kaiserschnittrate
}

\author{
Christiane Schwarz, Hebamme, MSc Public Health (MPH), Hannover
}

\section{Die neue Leitlinie der US-amerikanischen Geburtshelfer zur Senkung der Kaiserschnitt- rate hat das Potenzial, die geburtshilfliche Welt zu verändern. Man kann es kaum glauben: als im März 2014 die US-amerikanischen Geburtshelfer ihr Konsensuspapier zur Senkung der Kaiserschnittrate veröffentlichten, war es das erste Mal seit vielen
Jahren, dass von diesem Gremium selbstkritische Überlegungen zur medikalisierten
Geburtshilfe der vergangenen 20 Jahre laut wurden. Wir machen zu viele Kaiserschnitte
und wir retten damit weder Mütter noch Kinder [1], so der Tenor.}

Vorausgegangen war 2013 ein Editorial des Präsidenten der FIGO, Professor C. N. Purandare, der die Frage stellte, ob die aktuelle Geburtshilfe auf dem richtigen Weg sei [2]. Der steile Anstieg der Sectioraten in vielen Industrieländern werde begünstigt durch

- die einfacher werdende Operationstechnik (Misgav-Ladach-Methode),

- die Ausweitung von „weichen“ Indikationen (suspektes/pathologisches CTG, protrahierte Geburt/ Geburtsstillstand),

- finanzielle Fehlanreize,

- forensischen Druck und

- demografischen (Alter, Übergewicht) sowie sozialen (Wunschsectio) Veränderungen.

Die WHO stelle seit Jahren immer wieder fest, dass eine Sectio-Rate von über 15\% nicht medizinisch gerechtfertigt sei [3] und dass höhere Sectio-Raten nicht zu besseren perinatalen Ergebnissen führe [4]. Außerdem führen häufige primäre Sectiones zwangsläufig zu mehr Re-Sectiones [2].

\section{Ursachen und Auswirkun- gen unnötiger Sectiones}

Hierauf sattelt die US-amerikanische Fachgesellschaft für Gynäkologie und
Geburtshilfe (American College of Obstetricians and Gynecologists, ACOG) auf und veröffentlicht gemeinsam mit der Fachgesellschaft für Feto-Maternale Medizin (Society for Maternal-Fetal Medicine, SMFM) eine Stellungnahme mit Empfehlungen für die klinische Praxis, um unnötige Kaiserschnitte zu vermeiden.

Die Autoren stellen fest, dass der Kaiserschnitt in einigen Fällen eine sinnvolle Operation ist. Dazu gehören die Placenta praevia und die Uterusruptur. In anderen Situationen jedoch müssen die Vor- und Nachteile gut gegeneinander abgewogen werden. Für die weitaus meisten Geburten gilt, dass die Sectio im Vergleich zur vaginalen Geburt mit einem höheren Krankheits- und Sterberisiko für die Mutter verbunden ist (Tab. 1).

Dabei ist es schwierig, den ursächlichen Zusammenhang zwischen mütterlichen Problemen und der Sectio genau zu identifizieren. Trotz methodischer Schwächen diverser Studien lässt sich ein deutlicher Zusammenhang zwischen Sectio und Komplikationen nachweisen wie

- Atonie (mit und ohne Hysterektomie),

- Uterusruptur,

- Narkosezwischenfällen, Schock,

Herzstillstand, Atemstillstand,
- akutes Nierenversagen,

- Thromboembolien sowie

- Infektionen, Wundheilungsstörungen und Hämatomen.

Diese Probleme treten bei Frauen mit Sectio 3-mal häufiger auf im Vergleich zu Frauen, die vaginal geboren haben [5]. Dazu kommen Komplikationen in der Folgeschwangerschaft. So steigt die Wahrscheinlichkeit für eine Placenta praevia von $1 \%$ bei einem vorausgegangenem Kaiserschnitt auf 3\% oder höher, wenn die Frau bereits 3 Sectiones hatte. Außerdem liegt bei diesen Frauen das Risiko für eine Placenta praevia accreta bei $40 \%[6,7]$.

Die ACOG argumentiert, dass SectioRaten, die sich regional und nach Kliniken stark unterscheiden (sie liegen in den US-Bundesstaaten zwischen 23,1\% und $38,8 \%$ ) ein Hinweis darauf sein müssen, dass klinische Praxis und nicht medizinische Notwendigkeit Anlass für viele Sectiones sein müssen. Sehr deutlich wird dies bei der Spanne zwischen verschiedenen Kliniken: die niedrigste SectioRate in einer Klinik betrug im Untersuchungszeitraum bis 2011 gerade einmal $7,1 \%$, die höchste 69,9\%. Diese Varianz lässt sich durch die Unterschiede der Klientel (Alter, BMI, Morbidität und an- 
Tab. 1 Risiken in Abhängigkeit vom Geburtsmodus (modifiziert nach ACOG 2014).

\begin{tabular}{|l|l|l|}
\hline Risiken & vaginale Geburt & Sectio \\
\hline Mutter & & \\
\hline $\begin{array}{l}\text { gesamte schwere Morbidität/ } \\
\text { Mortalität }\end{array}$ & $8,6 \%$ & $9,2 \%$ \\
\hline Mütterliche Mortalität je 100000 & 3,6 & $2,7 \%$ \\
\hline Fruchtwasserembolie je 100000 & $3,3-7,7$ & 13,3 \\
\hline DR III/IV & $1-3 \%$ & 15,8 \\
\hline Plazentationsstörungen & Anstieg mit jeder vorausgegangenen Sectio \\
\hline Urininkontinenz & kein Unterschied 2 Jahre post partum \\
\hline Postpartale Depression & kein Unterschied & \\
\hline Kind & & kein Vorkommen \\
\hline Schnittverletzungen & kein Vorkommen & $1-2 \%$ \\
\hline Atemstörungen & $<1 \%$ & $1-4 \%$ \\
\hline Schulterdystokie & $1-2 \%$ & $($ bei prim. Sectio) \\
\hline
\end{tabular}

dere) nicht erklären. Es muss also - so die Autorinnen und Autoren - beeinflussbare Faktoren für die klinische Entscheidung zur Sectio geben [1].

\section{Vermeidung unnötiger Sectiones}

Im ACOG Konsensus werden die unterschiedlichen Faktoren beleuchtet, die zu Sectio-Entscheidungen führen können. Dabei spielt die Frage nach der Dauer einer normalen Geburt eine entscheidende Rolle: Wie lange dauern denn normale Geburten?

\section{Protrahierte Eröffnungsphase}

Seit den 1950er Jahren wird die Eröffnungsphase (EP) auf der Basis wissenschaftlicher Studien in eine latente und eine aktive Phase eingeteilt [8]. Damals galt eine Latenzphase (Zeit bis zur MMÖffnung von ca. $4 \mathrm{~cm}$ ) erst als ungewöhnlich lang, wenn sie länger als 20 Std. bei Erstgebärenden oder 14 Std. bei Mehrgebärenden dauerte. In der aktiven EP galt eine Eröffnungsgeschwindigkeit die Feststellung, dass die aktive Phase der Eröffnung vermutlich häufig erst ab $6 \mathrm{~cm}$ MM-Weite beginnt und somit auch der Zeitpunkt, ab dem ein protrahierter Geburtsverlauf erst diagnostiziert werden kann.

Mit der Feststellung, dass die meisten Frauen mit einer langen Latenzphase schließlich von selbst eine progressive Wehentätigkeit entwickeln, brauchen sehr wenige $\mathrm{zu}$ irgendeinem Zeitpunkt Wehenmittel. Eine lange Latenzphase (Zeit bis $6 \mathrm{~cm}$ MM-Weite) von mehr als 20 Std. (Erstgebärende) bzw. 14 Std. (Mehrgebärende) ist keine Indikation für einen Kaiserschnitt.

\section{Protrahierte Austreibungsphase}

Die Austreibungsphase (AP) beginnt bei vollständig eröffnetem Muttermund und endet mit der Geburt des Kindes. Wie lange diese dauert, wird von vielen Faktoren beeinflusst. Dazu gehören Parität, Zeitpunkt des Mitschiebens, Body-MassIndex der Mutter, Regionalanästhesie (1 Std. Geburtsdauer zusätzlich), Gewicht und Größe des Kindes sowie seine Einstellung und Haltung [10].

In Studien zu den Auswirkungen einer langen AP auf Mutter und/oder Kind konnten keine Zusammenhänge zwischen der Dauer der AP bei Erstgebärenden und gravierenden kindlichen Problemen (APGAR nach $5 \mathrm{~min} .<4$, arterieller NS-pH $<7,0$, Notwendigkeit zur sofortigen Intubation, Aufnahme auf die Kinderintensivstation, Sepsis) festgestellt werden, auch wenn die Austreibungsphase 3 Std. [11], 4 Std. [12] oder 5 Std. [13] gedauert hatte. Zu Mehrgebärenden liegt eine Studie vor, in der sich niedrigere APGAR-Werte und häufigere Verlegungen von Neugeborenen in die Intensivversorgung zeigten, wenn die AP länger als 3 Std. gedauert hatte [14]. Bei den Müttern zeigten sich häufiger ungünstige Auswirkungen, insbesondere häufiger schwere Dammverletzungen, Atonien und Infektionen [13]. 
Definition Geburtsstillstand in der Austreibungsphase

Eine „normale“ Dauer der AP ist nicht definierbar.

- Diagnose frühestens nach 3 Std. aktivem Mitschiebens bei Erstgebärenden oder 2 Std. bei Mehrgebärenden

- Bei PDA jeweils eine Stunde länger Ein Eingreifen ist auch nach diesem Zeitfenster nicht zwingend erforderlich!

Die Risiken einer langen AP sind jedoch überschaubar und nicht unbedingt vital bedrohlich; Komplikationen treten insgesamt selten auf und auch nach sehr langen Austreibungsperioden nicht deutlich häufiger als nach „normal“ langen Phasen $(<1,5 \%$ bei bis zu 2 Std. AP, $<3 \%$ nach 5 Std. und länger).

Daher empfiehlt die ACOG, in der aktiven AP (wenn die Frau mitschiebt) bei Erstgebärenden mindestens 3 und bei Mehrgebärenden 3 Std. abzuwarten, in Einzelfällen (z. B. bei PDA, bei großem Kind) auch deutlich länger [1].

\section{Vaginal-operative Geburten}

Während die Rate an sekundären Sectiones stark angestiegen ist, ist die Rate an vaginal-operativen Geburten (Vakuumextraktion, Forceps) in den letzten 15 Jahren stetig gesunken. Die Statistiken zeigen allerdings bei beiden Geburtsmodi (Sectio bzw. vaginal-operative Geburt) ähnliche Häufigkeiten neonataler Komplikationen wie Hirnblutungen oder Tod [15]. In einer neueren Studie gibt es sogar Hinweise auf ein selteneres Auftreten schwerer Komplikationen bei Forceps-Geburten im Vergleich zur sekundären Sectio oder Vakuumextraktion [16]. Somit ist die vaginal-operative Geburt eine sinnvolle mögliche Alternative zur sekundären Sectio.

Erfolg und Ergebnis der vaginal-operativen Geburtshilfe hängen von der
Situation (Höhenstand und Einstellung des Kopfes) und dem Geschick der Geburtshelfer ab.

Daher empfehlen die Autorinnen und Autoren, die notwendigen manuellen Fähigkeiten angemessen zu erlernen und zu üben [1].

\section{Fehleinstellungen}

Regelwidrige Einstellungen und Haltungen stehen im Zusammenhang mit sekundären Sectiones sowie mit neonatalen Komplikationen [17]. Eine Korrektur der Fehleinstellung durch manuelle Reposition und anschließender Rotation des Hinterkopfes kann in dieser Situation eine hilfreiche Intervention sein. In mehreren Studien konnte eine deutliche Reduzierung der Sectio-Raten in dieser Situation festgestellt werden $[18,19]$.

Bevor man sich für eine Sectio wegen Geburtsstillstand in der AP entscheidet, sollte eine sorgfältige Diagnose der fetalen Einstellung und Haltung gestellt und der Versuch einer manuellen Rotation erwogen werden.

\section{Pathologisches CTG}

Die zweithäufigste Indikation für eine sekundäre Sectio ist die Diagnose eines

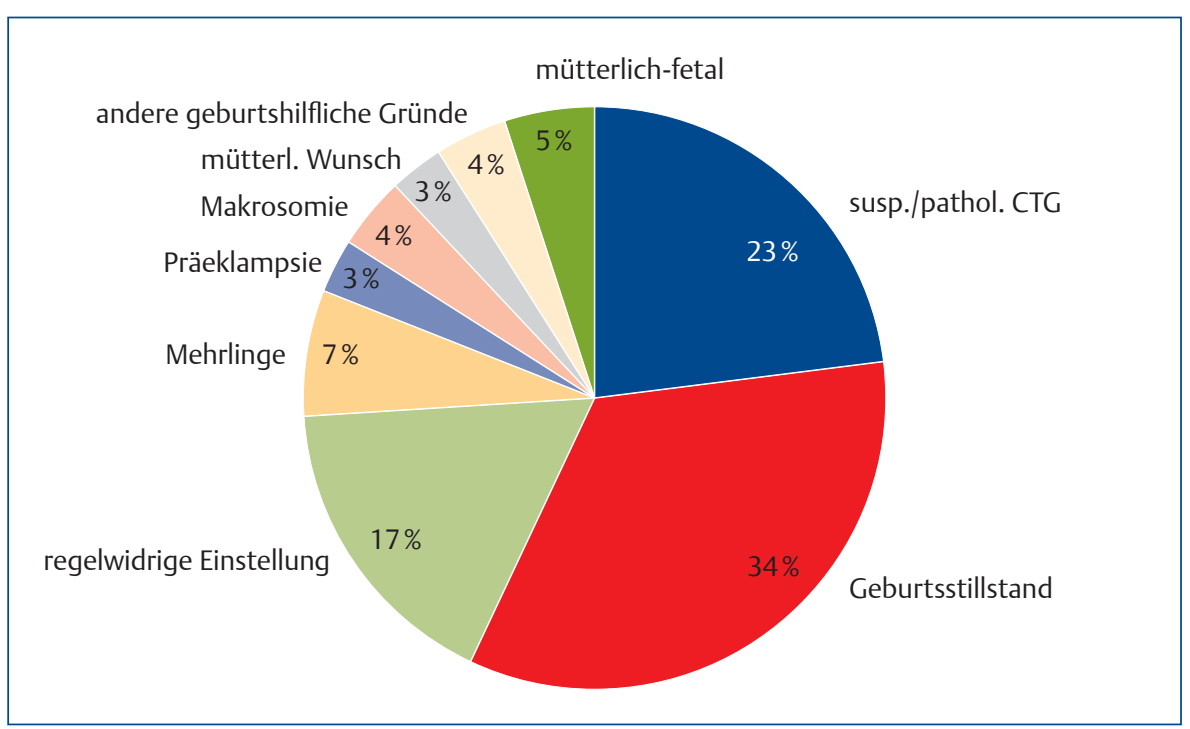

Abb. 1 Sectio-Indikationen in den USA (modifiziert nach ACOG 2014). suspekten oder pathologischen fetalen Herztonmusters (Abb. 1).

Hinsichtlich der Feststellung schwer pathologischer CTGs (nach FIGO: fehlende Oszillation, späte oder komplizierte Dezelerationen, Tachykardien, sinusoidale Muster und Bradykardien) besteht kaum Kontroverse. Diese Muster stehen im Zusammenhang mit niedrigen NSpH-Werten, aber auch mit Enzephalopathien [20]. Treten diese Muster auf, muss die Ursache gesucht, bewertet und wenn möglich behoben werden, ansonsten ist im Zweifelsfall eine Geburtsbeendigung angezeigt. Normale CTGs werden (unter der Voraussetzung, dass die fetalen und nicht die mütterlichen Herztöne abgeleitet werden) so gut wie nie mit vermeidbaren, schlechten fetalen Outcomes beobachtet. Auch hier ist die Handlungsempfehlung klar: es ist keinerlei Intervention nötig.

Das suspekte CTG, bei dem eine eindeutige Interpretation und Handlungsempfehlung schwierig ist, scheint als Anlass für eine sekundäre Sectio allerdings häufiger vorzukommen als das eindeutig pathologische CTG. Die Autorinnen und Autoren empfehlen, bei suspektem CTG zunächst Indizien für das fetale Wohlergehen zu suchen und konservative Korrektiva einzusetzen wie Flüssigkeitsgabe, Positionswechsel. Hier 
bietet sich als Alternative oder Ergänzung zur Mikroblutuntersuchung (MBU) die Stimulation des Feten (z. B. durch Berührung des Kopfes) an, denn spontane und stimulierte Bewegungen des ungeborenen Kindes korrelieren in der Regel mit guten postpartalen Ergebnissen [21, 22]. Daneben kann auch die Ergänzung des Fruchtwasservolumens mit Kochsalzlösung eine Verbesserung der fetalen Herztonmuster bewirken [23, 24]. Zusatzdiagnostik wie die Analyse der fetalen ST-Strecke im EKG (STAN) oder Puls-Oxymetrie sind nach Ansicht der Autorinnen und Autoren nur begrenzt geeignet, um die Interpretation von CTGs zuverlässiger zu machen [1].

Es gibt keine Nachweise, dass die gängigen Maßnahmen zur „intrauterinen Reanimation“ wie Sauerstoffgabe der Mutter, Infusionen und Tokolytika zu einem besseren Outcome der Neugeborenen führen.

\section{Geburtseinleitung}

Frauen, die eingeleitet werden, haben eine höhere Wahrscheinlichkeit, die Geburt per Sectio zu beenden. Es wurde lange angenommen, dass hier ein ursächlicher Zusammenhang besteht. Tatsächlich zeigen neuere Studien, dass die höhere Sectio-Rate eher mit der Ursache für die Einleitung im Zusammenhang steht und weniger mit der Einleitung selbst $[25,26,27]$. Die Autorinnen und Autoren verweisen auf eine viel diskutierte Übersichtsarbeit [28], in der eine generelle Geburtseinleitung ab der 41. vollendeten SSW empfohlen wird, da dies die perinatale Sterblichkeit senke. (siehe hierzu Stellungnahmen der DGHWi www.dghwi.de und der AWMF www.awmf.org)

In diesem Zusammenhang weisen die Autoren nachdrücklich darauf hin, dass bei einer Geburtseinleitung mit Interventionen in der Latenzphase zurückhaltend umgegangen werden soll.
Die Latenzphase kann hier länger als 24 Std. dauern und ein Wehentropf nach Blasensprung sollte mindestens 12-18 Std. wirken können, bevor ein eventueller Geburtsstillstand diagnostiziert wird [1].

\section{Beckenendlage}

Etwa 3,8\% aller Feten präsentieren sich bei Erreichen der 37. SSW in BEL. Von diesen Frauen entbinden über 85\% mit einem Kaiserschnitt. Die Autorinnen und Autoren verweisen darauf, dass die Möglichkeit der äußeren Wendung deutlich zu selten angeboten und durchgeführt wird [29].

\section{Verdacht auf Makrosomie}

Erst ein geschätztes fetales Gewicht ab $4500 \mathrm{~g}$ bei diabetischen Frauen und ab $5000 \mathrm{~g}$ bei nichtdiabetischen Frauen ist eine mögliche Indikation für einen Kaiserschnitt. 
Diese Einschätzung stützt sich auf die Berechnung einer „Number-Needed-toTreat" [30, 31]. Dabei wird berechnet, wie viele Behandlungen (hier: Sectio) durchgeführt werden müssen, um einmal den gewünschten Effekt (hier: Verhinderung von Schulterdystokie) zu erzielen. Anschließend kann die „Number-Neededto-Harm" berechnet werden, also die Anzahl von Behandlungen (hier: Sectio), die nötig ist, um einmal eine unerwünschte Nebenwirkung (hier: u.a. Atemstörungen des Neugeborenen) hervorzurufen. Nun kann man die Vor- und Nachteile einer Behandlung abwägen. Im Falle der Sectio überwiegen die Vorteile frühestens ab den o.g. geschätzten fetalen Gewichten.

\section{Zwillingsschwangerschaft}

Wenn das führende Kind in Schädellage liegt, verbessert sich das perinatale Outcome nicht durch einen geplanten Kaiserschnitt im Vergleich zu einer vaginalen Geburt.

Diesen Schwangeren sollte eine vaginale Geburt geraten werden [34].

\section{Herpes simplex-Infektion}

Lediglich bei aktivem Genitalherpes ist eine primäre Sectio indiziert. Bei Frauen mit positivem Befund in der Anamnese soll eine Prophylaxe mit Aciclovir durchgeführt werden. Anschließend kann eine vaginale Geburt angestrebt werden [35].

\section{Kontinuität der Betreuung}

Die effektivste Strategie, um die perinatalen Ergebnisse zu verbessern und damit auch die Sectio-Rate signifikant zu senken, ist die kontinuierliche Betreuung der Gebärenden [36].

Außerdem nimmt die Zufriedenheit der Frauen mit dem Geburtserleben in diesem Betreuungsmodell ebenfalls deutlich zu. Die ACOG stellt fest, dass diese
Tab. 2 Empfehlungen zur Prävention der primären Sectio (modifiziert nach ACOG 2014).

\begin{tabular}{|c|c|c|}
\hline & \multicolumn{2}{|c|}{ Empfehlungsgrad } \\
\hline & $\begin{array}{l}\text { Dringlich- } \\
\text { keit }\end{array}$ & $\begin{array}{l}\text { Evidenz- } \\
\text { level }\end{array}$ \\
\hline \multicolumn{3}{|l|}{ Eröffnungsperiode (EP) } \\
\hline $\begin{array}{l}\text { Eine protrahierte Latenzphase (Erstgebärende > } 20 \text { Std. Mehr- } \\
\text { gebärende > } 14 \text { Std.) ist keine Sectioindikation. }\end{array}$ & 1 & B \\
\hline $\begin{array}{l}\text { Eine langsame Eröffnungsgeschwindigkeit in EP ist keine } \\
\text { Sectioindikation. }\end{array}$ & 1 & B \\
\hline $\begin{array}{l}\text { Die MM-Weite von } 6 \mathrm{~cm} \text { sollte als Grenze zwischen Latenz- } \\
\text { phase und aktiver EP gelten. }\end{array}$ & 1 & B \\
\hline $\begin{array}{l}\text { Ein Geburtsstillstand in der EP sollte erst ab MM > } 6 \text { cm mit } \\
\text { offener Fruchtblase und regelmäßigen Wehen bei Status idem } \\
\text { für }>4 \text { Std. bzw. bei Status idem für }>6 \text { Std. mit Wehentropf } \\
\text { definiert werden. }\end{array}$ & 1 & B \\
\hline
\end{tabular}

\section{Austreibungsperiode (AP)}

Es gibt keine Definition zur normalen Dauer einer AP.

Geburtsstillstand in der AP sollte frühestens nach 2 Std.

(Mehrgebärende) bzw. 3 Std. (Erstgebärende) aktivem Mitschieben gestellt werden.

Eine längere AP ist u. U. physiologisch und erfordert nicht unbedingt ein Eingreifen.

Vaginal-operative Geburtshilfe durch erfahrene GynäkologInnen ist eine gute Alternative zur Sectio und sollte praktisch geübt werden.

Die Korrektur einer Fehleinstellung/Haltung durch manuelle Rotation des Kopfes kann eine sinnvolle Intervention sein, um eine sekundäre Sectio zu vermeiden.

\section{Fetale Überwachung}

Amnioinfusion bei variablen Dezelerationen

Stimulation am fetalen Skalp zum Ausschluss fetaler Azidose

\section{Geburtseinleitung}

Vor 41+0 SSW nur mit medizinischer Indikation, ab 41+0 SSW empfohlen

\begin{tabular}{|l|l|l|l|l|}
\hline Zervixreifung vor mechanischer Einleitung bei unreifem Befund & 1 & B
\end{tabular}

Latenzphasen von > 24 Stunden berücksichtigen

Bei offener Fruchtblase mindestens 12-18 Std. die Wirkung der Wehenmittelgabe abwarten

\section{Regelwidrige Einstellung/BEL}

genaue Diagnostik

bei BEL äußere Wendung empfehlen

\section{Verdacht auf Makrosomie}

Indikation zur Sectio erst ab mindestens $4500 \mathrm{~g}$ (Diabetes)

bzw. $5000 \mathrm{~g}$ (ohne Diabetes)

\section{Zwillingsschwangerschaft}

keine Sectioindikation, wenn der führende Zwilling in

Schädellage liegt 
Strategie, die außerdem nebenwirkungsfrei ist, vermutlich zu selten genutzt wird.

\section{Praktische Konsequenzen}

- Eine nationale Leitlinie kann dabei helfen, Maßnahmen zur Senkung der Sectio-Raten umzusetzen, aber das Umdenken muss auf allen Ebenen erfolgen: bei den Leistungserbringern, bei den Klinikleitungen, im Gesundheitssystem und bei den Frauen.

- Wirksame Mittel, um diese Herausforderung anzugehen, sind Praxisreflexion und die Erfassung und Auswertung von Qualitätsindikatoren, insbesondere, wenn diese Indikatoren verknüpft sind mit einem systematischen Vorgehen bei der Implementierung von Leitlinien. Chaillet et al. zeigten in einem Versuch in Kanada, dass die Sectio-Rate mit diesem Ansatz um 27\% gesenkt werden konnte [37].

- Ein ungelöstes Problem ist die Angst vor juristischen Konsequenzen, die zu unnötigen Interventionen - insbesondere Kaiserschnitten - anstiftet. Erst wenn sich Praktiker und Gutachter zu einer forschungsgestützten Entscheidungsfindung und in der Konsequenz interventionsärmeren Geburtshilfe durchringen können, wird sich dieses Problem im Laufe der Zeit nach entsprechenden Urteilen reduzieren.

\section{Literatur}

1 ACOG, 2014. Obstetric care consensus no. 1: safe prevention of the primary cesarean delivery. Obstetrics and gynecology; 123(3): 693-711

2 Purandare CN, 2013. Current obstetric practices: Are we on the right track? International journal of gynaecology and obstetrics; 123(2): 91-92

3 Chalmers B, 1992. WHO appropriate technology for birth revisited. British journal of obstetrics and gynaecology; 99(9): 709-710

4 Villar J et al., 2007. Maternal and neonatal individual risks and benefits associated with caesarean delivery: multicentre prospective study. BMJ (Clinical research ed.); 335(7628): 1025

5 Liu S et al., 2007. Maternal mortality and severe morbidity associated with low-risk planned cesarean delivery versus planned vaginal delivery at term. CMAJ: Canadian Medical Association journal; 176(4): 455-460

6 Silver RM, 2012. Implications of the first cesarean: perinatal and future reproductive health and subsequent cesareans, placentation issues, uterine rupture risk, morbidity, and mortality. Seminars in perinatology; 36(5): 315-323

7 Silver RM et al., 2006. Maternal morbidity associated with multiple repeat cesarean deliveries. Obstetrics and gynecology; 107(6): 1226-1232 
8 Friedman EA, 1972. An objective approach to the diagnosis and management of abnormal labor. Bulletin of the New York Academy of Medicine; 48(6): 842-858

9 Zhang J et al., 2010. Contemporary patterns of spontaneous labor with normal neonatal outcomes. Obstetrics and gynecology; 116(6): 1281-1287

10 Piper JM, Bolling DR, Newton ER, 1991. The second stage of labor: factors influencing duration. Am. J. of obstetrics and gynecology; 165 (4 Pt 1): 976-979

11 Le Ray C et al., 2009. When to stop pushing: effects of duration of secondstage expulsion efforts on maternal and neonatal outcomes in nulliparous women with epidural analgesia. Am. J. of obstetrics and gynecology; 201(4): 361.e1-7

12 Cheng YW, Hopkins LM, Caughey AB, 2004. How long is too long: Does a prolonged second stage of labor in nulliparous women affect maternal and neonatal outcomes? Am. J. of obstetrics and gynecology; 191(3): 933-938

13 Rouse DJ et al., 2009. Second-stage labor duration in nulliparous women: relationship to maternal and perinatal outcomes. Am. J. of obstetrics and gynecology; 201(4): 357.e1-7

14 Cheng YW et al., 2007. Duration of the second stage of labor in multiparous women: maternal and neonatal outcomes. Am. J. of obstetrics and gynecology; 196(6): 585.e1-6

15 Towner DR, Ciotti MC, 2007. Operative vaginal delivery: a cause of birth injury or is it? Clinical obstetrics and gynecology; 50(3): 563-581

16 Werner EF et al., 2011. Mode of delivery in nulliparous women and neonatal intracranial injury. Obstetrics and gynecology; 118(6): 1239-1246

17 Cheng YW et al., 2010. The association between persistent fetal occiput posterior position and perinatal outcomes: an example of propensity score and covariate distance matching. Am. J. of epidemiology; 171(6): 656-663

18 Shaffer BL et al., 2011. Manual rotation to reduce caesarean delivery in persistent occiput posterior or transverse position. The journal of maternalfetal \& neonatal medicine; 24(1): 65-72

19 McQuivey RW, 2013. Reichman O, Gdansky E, Latinsky B, Labi S, Samueloff A. „Digital rotation from occipito-poste- rior to occipito-anterior decreases the need for cesarean section". European journal of obstetrics, gynecology, and reproductive biology, 171(2), e3-4

20 ACOG, 2009. ACOG Practice Bulletin No. 106: Intrapartum fetal heart rate monitoring: nomenclature, interpretation, and general management principles. Obstetrics and gynecology; 114(1): 192-202

21 Elimian A, Figueroa R, Tejani N, 1997. Intrapartum assessment of fetal well-being: a comparison of scalp stimulation with scalp blood $\mathrm{pH}$ sampling. Obstetrics and gynecology; 89(3): 373-376

22 Clark SL, Gimovsky ML, Miller FC, 1984 The scalp stimulation test: a clinical alternative to fetal scalp blood sampling. Am. J. of obstetrics and gynecology; 148(3): 274-277

23 MacGregor SN et al., 1991. A prospective, randomized evaluation of intrapartum amnioinfusion. Fetal acid-base status and cesarean delivery. The Journal of reproductive medicine; 36(1): 69-73

24 Strong TH, 1995. Amnioinfusion. The Journal of reproductive medicine; $40(2)$ : 108-114

25 Stock SJ et al., 2012. Outcomes of elective induction of labour compared with expectant management: population based study. BMJ (Clinical research ed.); 344: e2838

26 Cheng YW et al., 2012. Induction of labor compared to expectant management in low-risk women and associated perinatal outcomes. Am. J. of obstetrics and gynecology; 207(6): 502.e1-8

27 Darney BG et al., 2013. Elective induction of labor at term compared with expectant management: maternal and neonatal outcomes. Obstetrics and gynecology; 122(4): 761-769

28 Gülmezoglu AM et al., 2012. Induction of labour for improving birth outcomes for women at or beyond term. The Cochrane database of systematic reviews, 6, p.CD004945

29 ACOG, 2006. ACOG Committee Opinion No. 340. Mode of term singleton breech delivery. Obstetrics and gynecology; 108(1): 235-237

30 Rouse DJ et al., 1996. The effectiveness and costs of elective cesarean delivery for fetal macrosomia diagnosed by ultrasound. JAMA; 276(18): 1480-1486

31 Rouse DJ, Owen J, 1999. Prophylactic cesarean delivery for fetal macrosomia diagnosed by means of ultrasonography-A Faustian bargain? Am. J. of obstetrics and gynecology; 181(2): 332-338

32 ACOG, 2013. ACOG Committee opinion no. 548: weight gain during pregnancy. Obstetrics and gynecology; 121(1): 210-212

33 Stotland NE, Hopkins LM, Caughey AB, 2004. Gestational weight gain, macrosomia, and risk of cesarean birth in nondiabetic nulliparas. Obstetrics and gynecology; 104(4): 671-677

34 Barrett JFR et al., 2013. A randomized trial of planned cesarean or vaginal delivery for twin pregnancy. The New England journal of medicine; 369(14): 1295-1305

35 ACOG, 2007. ACOG Practice Bulletin. Clinical management guidelines for obstetrician-gynecologists. No. 82 June 2007. Management of herpes in pregnancy. Obstetrics and gynecology; 109(6): 1489-1498

36 Hodnett ED et al., 2013. Continuous support for women during childbirth. The Cochrane database of systematic reviews, 7, p.CD003766

37 Chaillet N, Dumont A, 2007. Evidencebased strategies for reducing cesarean section rates: a meta-analysis. Birth: 34(1): 53-64

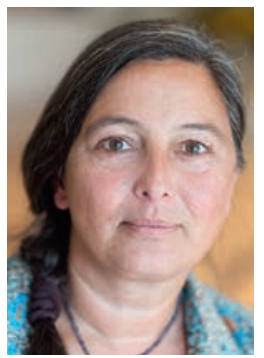

Christiane Schwarz

Hebamme, BSc Gesundheitswissenschaften, MSc Public Health (MPH), wissenschaftliche Mitarbeiterin an der Med. Hochschule Hannover Feldkamp 5, 31174 Schellerten E-Mail: christiane-schwarz@gmx.de 